\title{
ATLAS Soft (and some harder) QCD: Selected Results
}

\author{
RWL Jones ${ }^{1}$ for the ATLAS Collaboration \\ ${ }^{1}$ Department of Physics, Lancaster University, Lancaster LA1 4YB, UK
}

\begin{abstract}
Recent results on soft and hard QCD production are presented. Results derive from the $900 \mathrm{GeV}$ and 7,8 and $13 \mathrm{TeV}$ runs.
\end{abstract}

\section{Introduction}

ATLAS excels at high- $p_{T}$ physics, but also provides a window into important softer QCD processes. These have intrinsic interest but also provide the understanding that underpins the searches for new physics. ATLAS [1] now has data from $900 \mathrm{GeV}$ up to $13 \mathrm{TeV}$ in the same detector, allowing scale evolution to be probed.

The most relevant trigger and detector systems for the results presented here are the minimum bias trigger scintillators (MBTS), which have been refurbished for the $13 \mathrm{TeV}$ running (Run 2); and the inner detector, with good $p_{T}$ resolution, and excellent lifetime resolution; an Insertable B-Layer (IBL) [2] was added for Run 2, dramatically improving the lifetime resolution of the detector (from 100 to $50 \mathrm{fs}$ ) and enhancing the pattern recognition. The MBTS is made up of $2 \mathrm{~cm}$ thick disks of scintillator $3.6 \mathrm{~m}$ from the interaction region, giving a pseudorapidity coverage $2.07<|\eta|<3.86$. It has a high efficiency that is untiform to within $1 \%$.

The studies presented consider various classes of $p p$ interaction, and a glossary is needed. If both protons remain intact and there is no additional activity, the interaction is elastic. If one proton breaks up, it is single dissociation. If both break up, it is double dissociation. If the protons remain intact, but there is activity arising from pomerons or gluons exchanged between the two protons, there is central diffraction; and if there is the same activity accompanied by both protons breaking up, there is non-diffractive dissociation.

\section{Bose-Einstein Correlations}

This studies the correlations in phase space between two identical bosons arising from the symmetry of their wave functions. The enforced symmetry enhances likelihood of two particles being close in phase space, and allows one to probe the source of the bosons in size and shape. By investigating how the enhancement of close particle pairs depends on particle multiplicity and transverse momentum probes the production mechanism. The closeness of phase space is measured using the virtuality of particle pair, $Q^{2}=-\left(p_{1}^{2}-p_{2}^{2}\right)$, where $p_{i}$ is the particle 4-momentum. The strength of the effect is measured using a correlation function:

$$
C_{2}(Q)=N^{\operatorname{like} \operatorname{sign}_{(}}(Q) / N^{\mathrm{ref}}(Q)=c_{0}[1+\Omega(\lambda, Q R)](1+\epsilon Q),
$$


where $C_{0}$ is a normalisation factor, $\epsilon$ accounts for long-range effects, $R$ is the effective radius of the source and $\lambda$ is the strength of the effect, being 0 for a coherent source and 1 for a chaotic source. $N^{\text {like-sign }}(Q)$ is the number of like-sign particle pairs in a region of $Q$ and the reference sample $N^{\text {ref }}(Q)$ is the expected pair distribution without a Bose-Einstein effect. The reference sample can be formed using unlike sign particle pairs, particles from opposite hemispheres, or by mixing particles from different collisions. To remove the effect of resonances etc, the study uses the ratio

$$
R_{2}(Q)=C_{2}(Q) / C_{2}^{\text {Monte }} \operatorname{Carlo}(Q),
$$

where the Monte Carlo lacks Bose Einstein correlations. A current ATLAS study [4] sees a clear Bose-Einstein signal, and extends studies to very high charged particle multiplicity events (with > 108 charged particles reconstructed); this study uses oppositely charged particles (which are overwhelmingly pions). The fits work best assuming an exponential source, but results are also produced assuming a Gaussian source to compare with earlier measurements. Looking at the fitted source strength and radius as a function of multiplicity, both are seen to be essentially independent of centre of mass energy. However, the source radius growth saturates at high multiplicity as seen in figure 1, which is the first time that this has been observed. The behaviour is consistent with pomeron-based models.

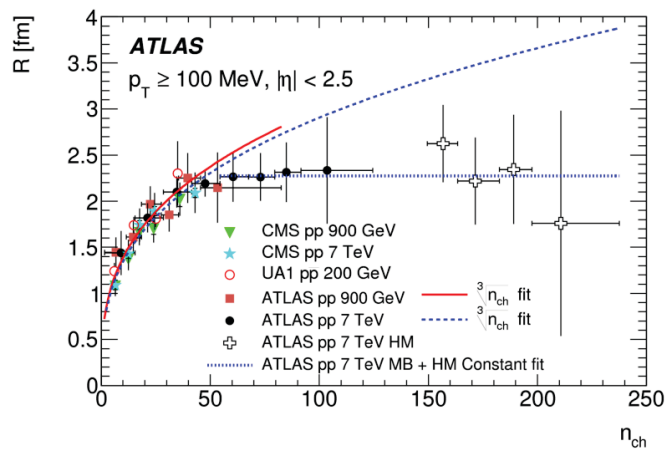

Figure 1. The source radius plotted as a function of event multiplicity [4].

The strength and radius can also be studied as a function of $k_{T}=\left|\bar{p}_{T, 1}+\bar{p}_{T, 2}\right| / 2$. This again shows no centre-of-mass energy dependence within the uncertainties, but when broken down by multiplicity region, the source radius decreases with increasing $k_{T}$, but increases with increasing multiplicity.

\section{Two Photon Scattering}

The LHC can be an effective two-photon collider. The predictions for the expected behaviour use the Equivalent Photon Approximation with absorbtive corrections for finite proton size. For the experimental study, the channel $\gamma \gamma \rightarrow l l$ is chosen [5]. There there is an elastic signal but dissociative backgrounds. The process $\gamma \gamma \rightarrow(W \rightarrow l v)(W \rightarrow l v)$ is the other important background. The signal selection uses the distance of dilepton from other activity (see figure 2 ) and the dilepton mass to discriminate from the background.

The experimental results using the $7 \mathrm{TeV}$ dataset improve on the precision of an earlier CMS result. Both in the electron and muon modes, the results agree with that earlier result, and with equivalent photon approximation predictions after absorptive corrections for finite proton size. 


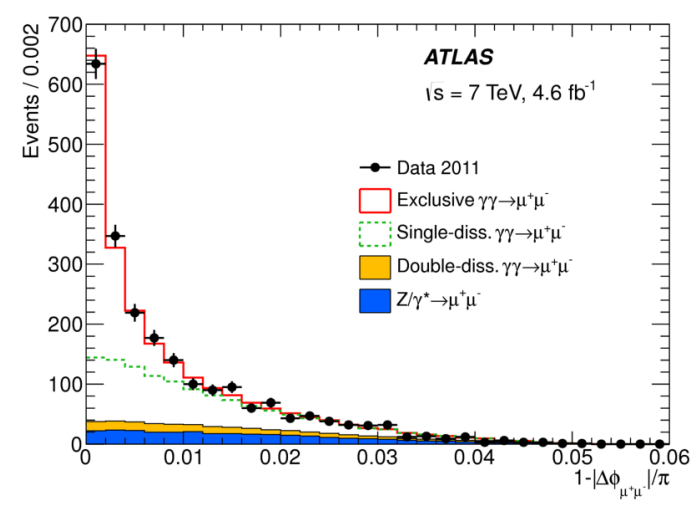

Figure 2. The acoplanarity in candidate $\gamma \gamma \rightarrow \mu^{+} \mu^{-}$events [5].

\section{Recent results on minimum bias events}

Unlike the harder collisions described above, soft particle production cannot be calculated from first principles. Instead, running models tuned to the data must be used. The tuning uses a wide range of measurements. Track multiplicities are now available up to $13 \mathrm{TeV}$ in ATLAS. The track-based analysis [6] uses dedicated runs with low pile-up of collisions in each readout of the detector, and events with only one reconstructed vertex are selected; the $13 \mathrm{TeV}$ sample had 10 million events, corresponding to an integrated luminoisty of $169 \mu \mathrm{b}^{-1}$. It also employs a special version of the track reconstruction that reconstructs tracks down to $100 \mathrm{MeV}$ momentum.

The differential cross-sections with respect to pseudorapidty $\eta$ and $p_{T}$ have been measured and compared to various models. Most models have a similar shape in $\eta$ but differ in normalisation. Herwig is the exception, and gives a poor agreement with the data; it is notable for having been tuned to the underlying event data. However, most models give a good agreement over 10 orders of magnitude in $p_{T}$, especially the EPOS model.

The charged particle multiplicity $\left(n_{c h}\right)$ distribution (within the $p_{T}$ and $\eta$ acceptances) have also been measured, and at low $n_{c h}$ no Monte Carlo model reproduces the data well. It is notable the models that lack colour reconnection (such as QGSJET) fail to reproduce the scaling with $n_{c h}$ seen in the data, see for example figure 3 .

The mean charged particle multiplicity $<n_{c h}>$ evolution with centre-of-mass energy $\sqrt{s}$ can also now be studied over a large energy range in a single experiment, and is well modelled except by HERWIG++.

\section{Recent results on the underlying event}

The underlying event is made up from all particles in the collision except those from the hard scatter. Its $\sqrt{s}$ dependence has also been examined with the new $13 \mathrm{TeV}$ data, and allows one to test the multiparton interaction modelling in various models. The technique used is to define a region sensitive to the underlying event in the plane perpendicular to the beam; this region is transverse to the direction of an object representing the hard scatter. The object used to define the hard scatter direction in the $7 \mathrm{TeV}$ data is either a $Z$ [7] or the leading jet [8]; for the $13 \mathrm{TeV}$ data it is the leading track [9]. Either the mean charged multiplicity or the mean scalar sum of particle $p_{T}$ in bins of pseudorapidity and angle to the leading object can be studied. These can be studied as a function of the $p_{T}$ of the leading 


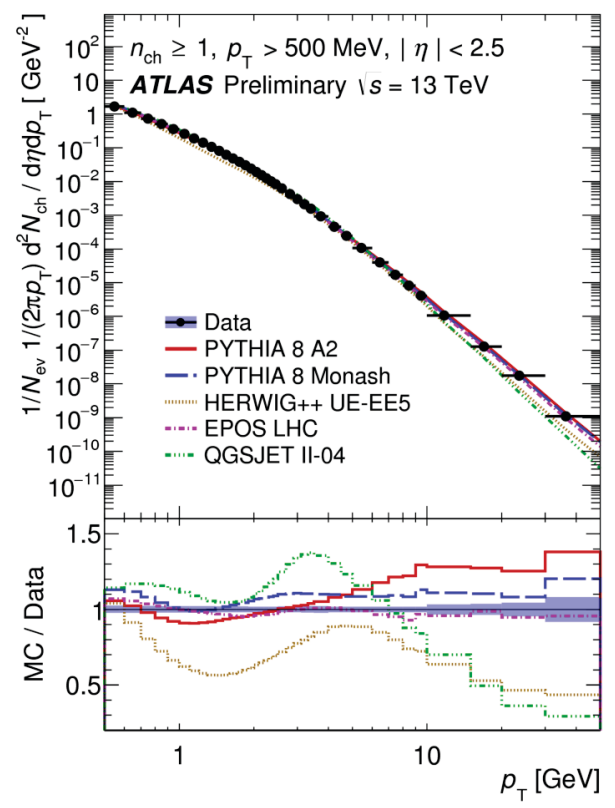

Figure 3. The multiplicity in minimum bias events as a function of pseudorapidity and track $p_{T}$ [6].

object. The data are broadly compatible between hard object definitions, particularly at high- $p_{T}$ of the leading object. The $Z$ and jet measures agree particularly well at high $p_{T}$, while at low $p_{T}$ the selections for the $Z$ introduce biases.

For the $13 \mathrm{TeV}$ data, the uncorrected mean scalar sum of particle $p_{T}$ and $n_{c h}$ have been studied as a function of angle to the leading object. PYTHIA tuned to the minimum bias data agrees well for a leading object $p_{T}>1 \mathrm{GeV}$, while the best agreement is with Monte Carlos tuned to lower energy underlying event data for leading $P_{T}>5 \mathrm{GeV}$. The mean charged multiplicity and scalar sum of particle $p_{T}$ has also been studied in the transverse and 'toward' (i.e. around the leading object) regions. It is hard for models to simultaneously describe the results for these two quantities, and (in contrast to the minimum bias case) EPOS gives a poor description of the data.

\section{Inelastic cross-section measurements}

The total inelastic cross-section can be determined using two distinct methods. The first uses the optical theorem to relate the elastic events measured in the ALFA Roman pot detectors to the inelastic cross section [10]. The other, new, result counts the inelastic events triggering the MBTS directly with a large rapidity gap [11]. The mass of the largest of the two jets triggering the MBTS is calculated; the coverage of the MBTS imposes a fiducial requirement that this is greater than $13 \mathrm{GeV}$. Again, low pile-up data is used. The fraction of event with only hits on one side of the MBTS is used to constrain the diffractive contribution. The fiducial cross-section is found to be $65.2 \pm 0.8$ (exp.) \pm 5.9 (lum.)mb, where the luminosity uncertainty dominates. The models tend to predict a slightly higher cross-section but largely agree within the uncertainties. 


\section{Jet results}

Many results on jets, usually using the anti-kT algorithm with a cone size $R=0.4$ are now available for the $13 \mathrm{TeV}$ results [12]. A cross-section for jets with $|y|<0.5$ has been measured and found to be in good agreement with Next to Leading Order calculations for various parton distribution functions. Energy-energy calculations have also been used to obtain a determination of the strong coupling [13] that is in good agreement with measurements from many other processes as shown in figure 4 . Run to the $Z$ pole, the result is $\alpha_{s}\left(m_{Z}\right)=0.1173 \pm 0.0010(\exp .)_{-0.0020}^{+0.006}$ (scale) $\pm 0.0017(\mathrm{PDF}) \pm 0.0002(\mathrm{NPC})$, where the last term describes the uncertainties from non-perturbative corrections.

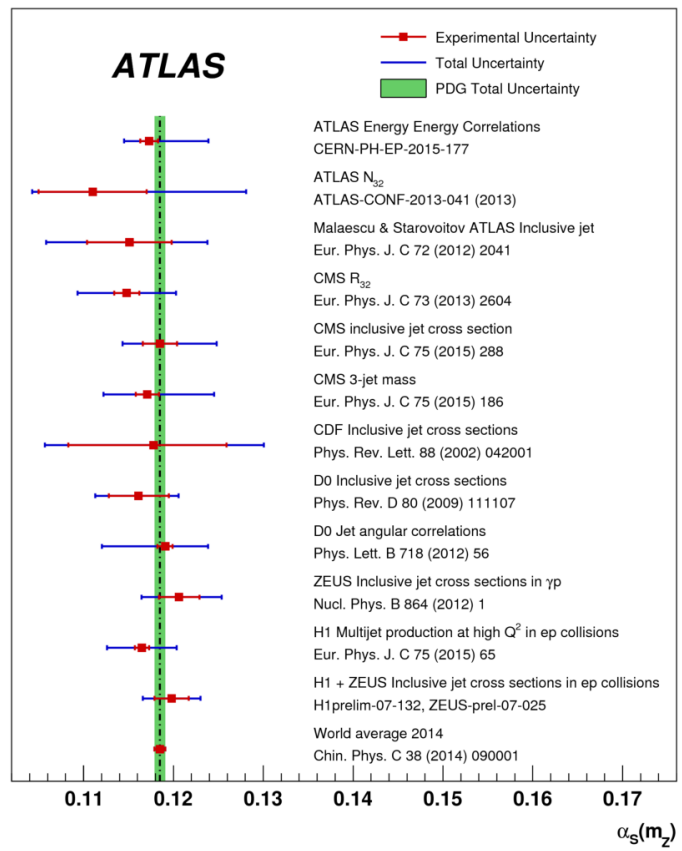

Figure 4. The strong coupling determined from energy-energy correlations in ATLAS compared to determinations in other processes; the plot is from the additional material provided for reference [13], and can be found at https://atlas.web.cern.ch/Atlas/GROUPS/PHYSICS/PAPERS/STDM-2014-03/\#auxstuff

\section{References}

[1] ATLAS Collaboration, JINST 3, S08003 (2008)

[2] ATLAS IBL Collaboration, CERN-LHCC-2010-013 (2010) https://cds.cern.ch/record/1291633; ATLAS Collaboration, CERN-LHCC-2010-013 (2013) https://cds.cern.ch/record/1291633

[3] ATLAS TDAQ Collaboration, JINST 9, C10020 (2014)

[4] ATLAS Collaboration, Eur. Phys. J. C75, 466 (2015)

[5] ATLAS Collaboration, Phys. Lett. B749, 242 (2015)

[6] ATLAS Collaboration, CERN-EP-2016-014 (2015) arXiv:1602.01633 [hep-ex]

[7] ATLAS Collaboration, Eur. Phys. J. C74, 3195 (2014) 
[8] ATLAS Collaboration, Eur. Phys. J. C74, 2965 (2014)

[9] ATLAS Collaboration, ATL-PHYS-PUB-2015-019 (2015) https://cds.cern.ch/record/2037684 [10] ATLAS Collaboration, Nucl. Phys. B, 486 (2014)

[11] ATLAS Collaboration, ATLAS-CONF-2015-038 (2015) https://cds.cern.ch/record/2045064

[12] ATLAS Collaboration, ATLAS-CONF-2015-034 (2015) https://cds.cern.ch/record/2038145

[13] ATLAS Collaboration, Phys. Lett. B750 427 (2015) 